\title{
miRNA Regulation Network Analysis in Qianliening Capsule Treatment of Benign Prostatic Hyperplasia
}

\author{
Liya Liu,, ${ }^{1,2}$ Yun Wan, ${ }^{1,2}$ Aling Shen,,2 Jinyan Zhao, ${ }^{1,2}$ Jiumao Lin,, Xiaoyong Zhong, \\ Yuchen Zhang, ${ }^{1,2}$ and Zhenfeng Hong ${ }^{1,2}$ \\ ${ }^{1}$ Academy of Integrative Medicine Biomedical Research Center, Fujian University of Traditional Chinese Medicine, \\ Fuzhou, Fujian 350122, China \\ ${ }^{2}$ Fujian Key Laboratory of Integrative Medicine on Geriatrics, Fujian University of Traditional Chinese Medicine, \\ Fuzhou, Fujian 350122, China
}

Correspondence should be addressed to Zhenfeng Hong; zfhong1953@163.com

Received 22 January 2015; Revised 5 April 2015; Accepted 8 April 2015

Academic Editor: Claudia Di Giacomo

Copyright (C) 2015 Liya Liu et al. This is an open access article distributed under the Creative Commons Attribution License, which permits unrestricted use, distribution, and reproduction in any medium, provided the original work is properly cited.

\begin{abstract}
Objective. The objective of this study was to evaluate the molecular mechanism by which Qianliening capsule (QC) treats benign prostatic hyperplasia (BPH). Methods. Benign prostatic hyperplasia epithelial cell line BPH-1 was treated with 0, 1.25, 2.5, and $5 \mathrm{mg} / \mathrm{mL}$ QC for $48 \mathrm{~h}$, respectively. Evaluation of cell viability and observation of morphologic changes of BPH-1 cell gene expression and miRNA expression profiles were analyzed. Real-time quantitative PCR was used to confirm changes in miRNA and gene expression. GO and KEGG pathway-based approaches were used to investigate biological functions and signaling pathways affected by differentially expressed mRNAs. Results. QC inhibited BPH-1 cell proliferation. Differential expression of 19 upregulated and 2 downregulated miRNAs was observed in QC-treated BPH-1 cells compared to untreated control cells. 107 upregulated and 71 downregulated genes were identified between the two groups. Significantly enriched signaling pathways based on deregulated mRNAs were mainly involved in regulation of cell proliferation, apoptosis, and so on. Additionally, miRNA-mRNA network analysis integrated these miRNAs and genes by outlining interactions of miRNA and related genes. Conclusion. The study was the first report of differentially expressed miRNA and mRNA in QC-treated BPH-1 cells.
\end{abstract}

\section{Introduction}

Benign prostatic hyperplasia $(\mathrm{BPH})$ is a pathological overgrowth of the human prostate, a condition that affects a majority of men older than 50 years [1]. Most patients with $\mathrm{BPH}$ experience increased resistance in urinary flow leading to urinary tract symptoms (LUTS) including urinary hesitancy, frequent urination, urgency, thin urine flow, and bladder stones [2]. These symptoms greatly affect the physical and mental health of patients as well as their quality of life. Unfortunately, the cellular and molecular mechanisms contributing to $\mathrm{BPH}$ development and progression remain unclear.

Microribonucleic acid (miRNA), a 19-24 noncoding family of nucleotide (nt), is processed from 70 to $100 \mathrm{nt}$ double-stranded hairpin precursors by RNase III dicer and endogenously expressed in the RNA-induced silencing complex within the cytoplasm [3]. miRNA recognizes the $3^{\prime}$ untranslated region of target $\mathrm{mRNA}(\mathrm{s})$ with imperfect complementarity which causes translational repression or mRNA cleavage [4]. Currently, growing evidence highlights the importance of miRNAs in association with pathophysiologic processes including abnormal cell proliferation and differentiation, invasion, metastasis, and the poor prognosis of various cancers and relative diseases [5-9]. Therefore, it is plausible that certain miRNAs participate in the pathogenesis of $\mathrm{BPH}$, which constitutes the rationale for our research.

The mainstay of pharmacotherapy for $\mathrm{BPH}$ is a combination treatment of $5 \alpha$-reductase inhibitors that regulate levels of 5-dihydrotestosterone (DHT) and $\alpha$-adrenergic blockers that inhibit $\alpha$-adrenergic receptors. However, these 
medications may induce undesired side effects such as orthostatic hypotension, decreased libido and ejaculation, or erectile dysfunction [10-14]. Qianliening capsule (QC) is a traditional Chinese medicine formulation consisting of wine rhubarb, leech, Milkvetch root, Achyranthes aspera, and dodders. These components together confer QC properties of heat-clearing, detoxification, promotion of blood circulation, removal of blood stasis, tonifying the kidney, and nourishing vitality (replenishing the kidney qi in Chinese) [15, 16]. Previous studies have demonstrated that QC has significant therapeutic effects on BPH [16-18]. In clinical trials, QC clearly improved a series of lower urinary tract symptoms (LUTS) in BPH patients, such as frequency of urination, urinary urgency, thin urine flow, incontinence, and other voiding disorders [19]. Our preliminary study in a rat model of BPH showed that QC significantly decreased prostatic volume and weight, inhibited prostatic hyperplasia, attenuated abnormal serum levels of estrogen and androgen, regulated the expression of estrogen receptor (ER), androgen receptor (AR), and related mRNA, inhibited the EGF/STAT3 pathway, and reduced expression of proproliferative PCNA, cyclin D1, and CDK4 proteins [15-19]. Moreover, QC effectively inhibited proliferation and promoted apoptosis in human benign prostatic hyperplasia epithelial cells and prostate cells $[20,21]$. To more fully clarify the mechanistic effects of $\mathrm{QC}$ therapy in the treatment of $\mathrm{BPH}$, we performed the present study to examine the effects of QC on expression of specifically expressed miRNAs, genes, and relevant signaling pathways in our rat model of BPH.

\section{Materials and Methods}

2.1. Materials and Reagents. Fetal bovine serum (FBS), Roswell Park Memorial Institute 1640 (RPMI 1640) medium, penicillin-streptomycin, and trypsin-EDTA were purchased from Life Technologies (Carlsbad, CA, USA). A cell proliferation WST-1 assay kit was purchased from Roche Applied Science Gmbh (Mannheim, Germany). TRIzol reagent was purchased from Life Technologies (Carlsbad, CA, USA). Superscript II reverse transcriptase was obtained from the Promega Corporation (Madison, WI, USA). All other chemicals, unless otherwise stated, were obtained from SigmaAldrich (St. Louis, MO, USA).

2.2. Preparation of QC. Qianliening capsule (QC, Fujian, China, FDA approval number: Z09104065) was provided by the Academy of Pharmacology of Fujian Chinese Medical University. The drug powder inside the capsule was dissolved in distilled water and stored at $-20^{\circ} \mathrm{C}$. Working concentrations of QC were prepared by diluting the stock solution in culture medium.

2.3. Cell Culture. Benign prostatic hyperplasia epithelial (BPH-1) cells were obtained from Xiangya Cell Center, University of Zhongnan (Hunan, China), and grown in RPMI 1640 medium. RPMI 1640 was supplemented with 10\% (v/v) FBS and 100 units $/ \mathrm{mL}$ penicillin and $100 \mu \mathrm{g} / \mathrm{mL}$ streptomycin. All cells were cultured at $37^{\circ} \mathrm{C}$ and $5 \% \mathrm{CO}_{2}$ under humidified environment.
2.4. Evaluation of Cell Viability by WST-1 Assay. The influence of increasing QC concentration on the viability of BPH-1 cells was determined using a cell proliferation reagent WST1 kit. Briefly, BPH-1 cells were harvested from exponential phase cultures growing in RPMI 1640 with $10 \%$ FBS, counted, plated in 96-well flat-bottomed microtiter plates $(100 \mu \mathrm{L}$ cell suspensions, $2.5 \times 10^{4}$ cells $/ \mathrm{mL}$ ), and treated with medium containing various concentrations $(0 \mathrm{mg} / \mathrm{mL}, 1.25 \mathrm{mg} / \mathrm{mL}$, $2.5 \mathrm{mg} / \mathrm{mL}$, and $5 \mathrm{mg} / \mathrm{mL}$ ) of QC. After $48 \mathrm{~h}, 10 \mu \mathrm{L}$ WST-1 added to each well and the reaction mixture was incubated at $37^{\circ} \mathrm{C}$ in a $5 \% \mathrm{CO}_{2}$ atmosphere for $0.5 \mathrm{~h}$. Sample absorption was measured under a wavelength of $450 \mathrm{~nm}$ using a spectrophotometer (Bio Tek Model ELX800, USA), and the results were compared as percentages of control cells.

2.5. Observation of Morphologic Changes. BPH-1 cells were seeded into 6 -well plates at a density of $1.0 \times 10^{5}$ cells/well in $2 \mathrm{~mL}$ medium. The cells were treated with various concentration $(0,1.25,2.5,5 \mathrm{mg} / \mathrm{mL})$ QC for $48 \mathrm{~h}$. Cell morphology was observed using a phase-contrast microscope (Leica, Mannheim, Germany). All images were acquired at 400x magnification.

2.6. RNA Extraction for Microarray and Real-Time PCR Analysis. BPH-1 cells were seeded into $75 \mathrm{~cm}^{2}$ flasks at a density of $5 \times 10^{5}$ cells/flask in $15 \mathrm{~mL}$ medium. The cells were treated with $2.5 \mathrm{mg} / \mathrm{mL} \mathrm{QC}$ for $48 \mathrm{~h}$ and total RNA, including small RNA, was isolated with TRIzol reagent. RNA purity and concentration were determined via OD 260/280 readings using a spectrophotometer (NanoDrop 2000c). Concerning differential miRNAs and mRNAs with comparative microarraydetermined expression levels over 2.0 or below 0.5 , realtime PCR assays were utilized to detect and quantify the differential pre-miRNAs and mRNAs as previously described $[22,23]$ using SYBR Green dye. U6 was used as an internal control. The threshold cycle (CT) is defined as the fractional cycle number at which fluorescence passes a fixed threshold. The miRNA and mRNA expression levels were normalized to U6, relative expression was calculated using the comparative $\Delta \Delta \mathrm{CT}$ method, and values were expressed as $2^{-\Delta \Delta \mathrm{CT}}[24]$.

2.7. miRNA Expression Microarray Analysis. A GeneChip miRNA 3.0 array (Affymetrix, Santa Clara, CA) was hybridized using $500 \mathrm{ng}$ total RNA per standard Affymetrix protocols. The same RNA preparations used in the mRNA microarray analysis were used for miRNA array analysis. Data extraction was completed using Affymetrix Command Console software. Raw data was analyzed by the following workflow: background detection, RMA global background correlation, quantile normalization, median polish, and $\log 2$ transformation with miRNA QC tool software.

2.8. Gene Expression Microarray Analysis. Microarray analysis was accomplished by hybridization to a GeneChip PrimeView human gene expression array per manufacturer's instructions (Affymetrix, Santa Clara, CA). Genes that underwent at least a 2 -fold change between treatment groups were selected for real-time PCR validation. 


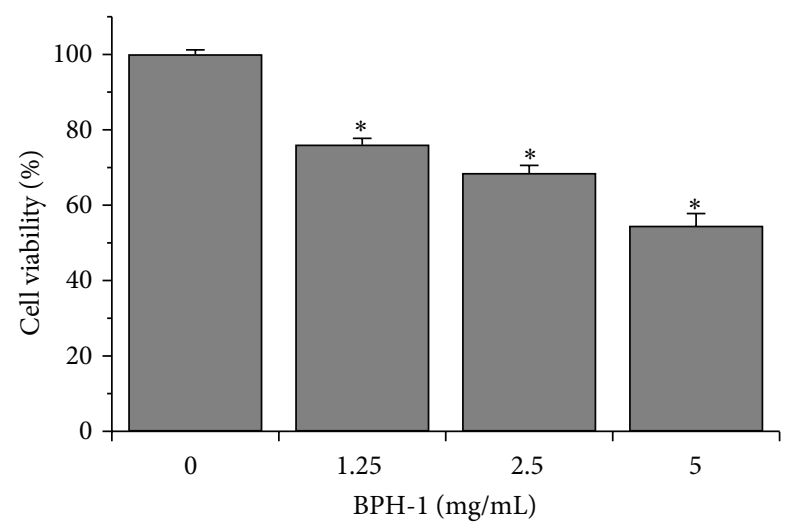

FIGURE 1: Effect of QC on BPH-1 cell viability. Cell viability was determined by the WST-1 assay after BPH-1 cells were treated with various concentrations of QC for $48 \mathrm{~h}$. The data were normalized to the viability of control cells $(100 \%)$. Data are the averages with SD (error bars) from 3 independent experiments. ${ }^{*} P<0.05$, versus control cells.

2.9. Gene Ontology and Kyoto Encyclopedia of Genes and Genomes Pathway Analysis Based on Differentially Expressed mRNAs. Gene ontology (GO) (http://www.geneontology .org/) and Kyoto Encyclopedia of Genes and Genomes (KEGG) databases (http://www.genome.ad.jp/kegg/) were used to investigate biological functions and signaling pathways affected by differentially expressed mRNAs [25-27]. A $P$ value less than 0.05 was considered statistically significant. Meanwhile, we constructed the miRNA-mRNA regulatory network basis on different miRNAs and their targets; the miRNA-mRNA interaction network, representing critical miRNAs and their targets, was established according to miRNA degree [28]. Networks of miRNA and genes from differentially expressed miRNA and mRNA in the two groups were visualized with the Cytoscape tool [28].

2.10. Statistical Analysis. Data are presented as means \pm SD for the indicated number of independently performed experiments and analyzed using the SPSS software package for Windows (version 16.0). Statistical analysis of the data was performed with Student's $t$-test and ANOVA. Differences with $P<0.05$ were considered to be statistically significant.

\section{Results}

3.1. QC Inhibits Proliferation of BPH-1 Cells. The effect of QC on BPH-1 cell viability was determined by the WST-1 assay. As shown in Figure 1, treatment with $1.25-5 \mathrm{mg} / \mathrm{mL}$ QC for $48 \mathrm{~h}$ reduced cell viability by $50 \%-80 \%$ in a dosedependent manner compared with untreated control cells $(P<0.05)$. To further verify these results, we evaluated the effect of QC on BPH-1 cells confluence via phase-contrast microscopy. QC treatment gradually decreased monolayer confluence with increasing drug concentration (Figure 2). Taken together, these data demonstrate that QC inhibited $\mathrm{BPH}-1$ cell proliferation.
TABLE 1: Differentially expressed miRNA between the BPH-1 cells treated with QC $(2.5 \mathrm{mg} / \mathrm{mL})$ group and control group.

\begin{tabular}{lc}
\hline miRNA & Fold change (QC versus CTR) \\
\hline miR-3663-3p & 5.25562 \\
miR-4730 & 5.15551 \\
miR-34c-5p & 3.4525 \\
miR-122 & 3.40821 \\
miR-4674 & 3.30796 \\
miR-34a & 3.28902 \\
miR-4734 & 3.19241 \\
miR-3178 & 3.1713 \\
miR-4497 & 3.164 \\
miR-4649-5p & 2.96402 \\
miR-3185 & 2.96402 \\
miR-3621 & 2.90446 \\
miR-663 & 2.60666 \\
miR-4634 & 2.55855 \\
miR-4745-5p & 2.31046 \\
miR-34a & 2.21767 \\
miR-4665-5p & 2.11388 \\
miR-3195 & 2.06861 \\
miR-1231 & 2.04573 \\
miR-125b-1 & -2.47347 \\
miR-1972 & -2.76246 \\
\hline
\end{tabular}

Positive value indicated upregulation and negative value indicated downregulation. miRNA with expression fold change $>2$ and $P$ value $<0.05$ was considered statistically significant. QC: BPH-1 cells treated with QC $(2.5 \mathrm{mg} / \mathrm{mL})$; CTR: control group.

3.2. miRNA Expression Profiling. The GeneChip miRNA array version 3.0 (Affymetrix) was used to profile miRNAs that were differentially expressed between $\mathrm{BPH}-1$ cells treated with QC $(2.5 \mathrm{mg} / \mathrm{mL})$ and control cells. All of the 1733 miRNAs that were probed, 21 were differentially expressed in QC-treated BPH-1 cells (Table 1). These are shown visually in a cluster analysis (Figure 3(a)). 19 of 21 were overexpressed whereas 2 of 21 were underexpressed. The miR-34 family (including miR-34a and miR-34c), miR-3185, miR-663, and miR-125b, are involved in apoptosis and proliferation [2934]; miR-122 and miR-1231 are associated with liver disease [35-37], and miR-3663 is associated with skin or smooth muscle cancer $[38,39]$. However, the biological functions of the other miRNAs that significantly changed in the miRNA array have not been reported previously and require further study.

3.3. Gene Expression Profiling. To gain insight into the mechanisms of QC action, we conducted gene expression profiling by GeneChip PrimeView human gene expression array, as shown visually via cluster analysis (Figure 3(b)). Significant changes in 178 genes were observed between QC-treated $\mathrm{BPH}-1$ cells and controls, of which 107 genes were upregulated and 71 genes were downregulated (Table 2). Genes that were identified as differentially expressed exhibited $>2.0$-fold change between the BPH-1 cells treated with QC and control cells. 

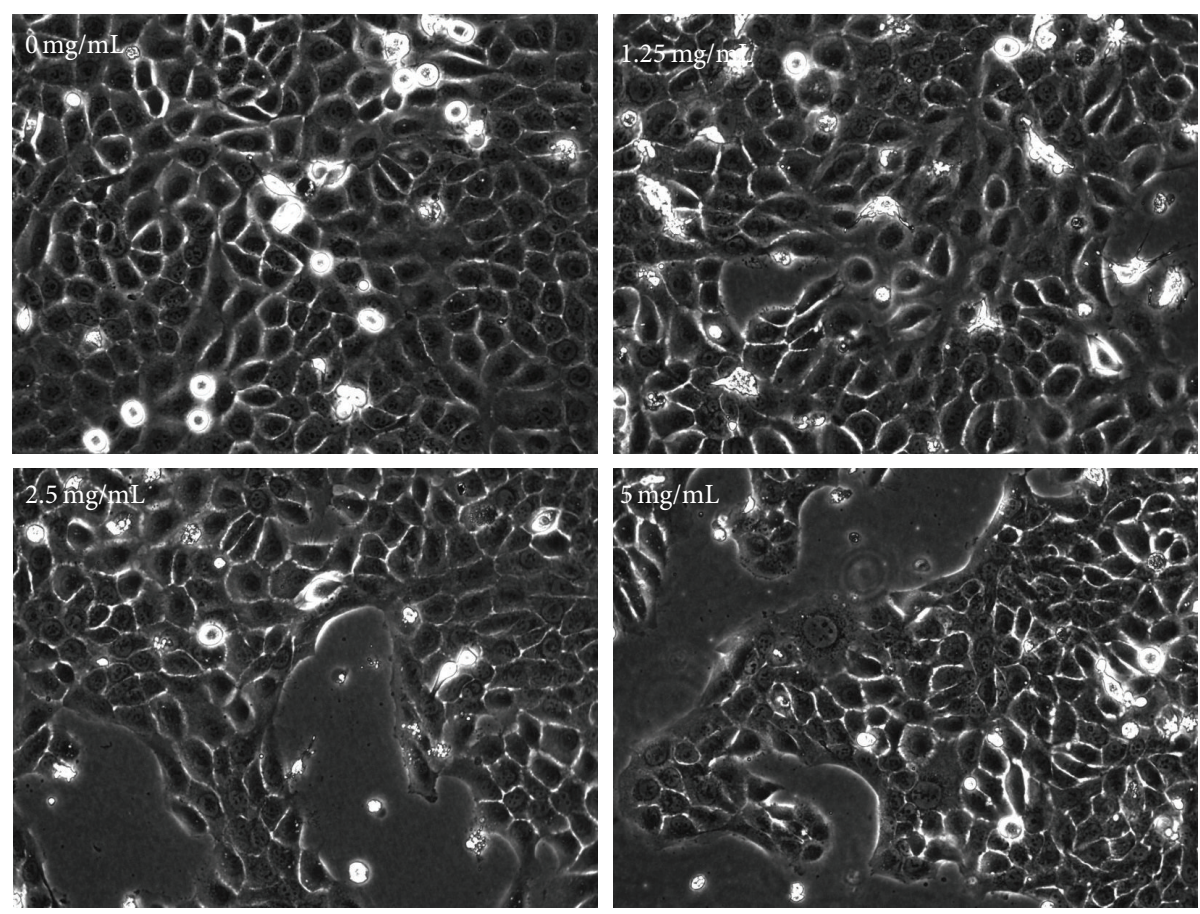

FIGURE 2: QC treatment and BPH-1 cell confluence. QC treatment dose-dependently decreased the cell density of BPH-1 cells, which was observed via phase-contrast microscopy. Images are representatives of 3 independent experiments.

TABLE 2: Differentially expressed genes between the BPH-1 cells treated with QC $(2.5 \mathrm{mg} / \mathrm{mL})$ group and control group.

\begin{tabular}{lc}
\hline Genes & Fold change (QC versus CTR) \\
\hline CYP1A1 & 12.8207 \\
CYP1B1 & 8.49316 \\
ALDH3A1 & 8.25975 \\
AKR1C1 & 6.11892 \\
AKR1C3 & 4.37771 \\
EDIL3 & 3.87485 \\
SCGB1A1 & 3.78913 \\
SEMA5A & 3.39468 \\
DOCK8 & 3.31441 \\
MPPED2 & 3.14276 \\
SPINK6 & -5.87906 \\
KRTAP2-3 & -4.78595 \\
ANKRD1 & -4.25824 \\
S1PR1 & -3.49797 \\
SPINK7 & -3.34239 \\
DUSP6 & -3.24443 \\
CA9 & -3.20190 \\
PTGS2 & -3.06597 \\
BNIP3 & -2.82441 \\
IL7R & -2.81757 \\
\hline
\end{tabular}

Positive value indicated upregulation and negative value indicated downregulation. Genes expression fold change $>2$ and $P$ value $<0.05$ were considered statistically significant. QC: BPH-1 cells treated with QC $(2.5 \mathrm{mg} / \mathrm{mL})$ group; CTR: control group. The top 10 different genes are shown.
3.4. Validation of Differentially Expressed miRNAs and Genes. Real-time PCR was used to validate the microarray analysis findings. The relative expressions, expressed as percent change from controls of the five chosen miRNAs (miR-34a, miR-122, miR-34c, miR-4703, and miR-1972), are shown in Figure 4(a). These data confirmed that miR-34a, miR-122, miR-34c, and miR-4703 were increased in QC-treated BPH1 cells whereas miR-1972 expression was decreased $(P<$ 0.05 for all). Meanwhile, we randomly selected 8 different genes (CYP1B1, ALDN3, CYP1A1, ANKRD1, KRTAP2-3, and SPINK2), and these data confirmed that CYP1B1, ALDN3, CYP1A1, and ANKRD1 were overexpressed in two groups whereas KRTAP2-3 expression was decreased $(P<0.05$ for all) (Figure 4(b)). SPINK2 expression, which was not significantly different from controls, was also confirmed not to be significantly different between the two groups.

3.5. GO and KEGG Pathway Analysis of Deregulated mRNAs. GO analysis showed that the differentially expressed mRNAs between QC-treated BPH-1 cells and the control group were significantly enriched in oxidation reduction, regulation of cell proliferation, immune response, regulation of apoptosis, regulation of cell death, and so forth. KEGG pathway analysis indicated that the deregulated mRNAs between the two cell groups were mainly involved in metabolism of xenobiotics by cytochrome P450, steroid hormone biosynthesis, drug metabolism, and TGF-beta signaling pathway ( $P$ value $<0.05$ after multiple testing corrections) (Tables 3-4). Additionally, miRNA-mRNA network analysis integrated these miRNAs and genes by outlining the interactions between them (Figure 5). 


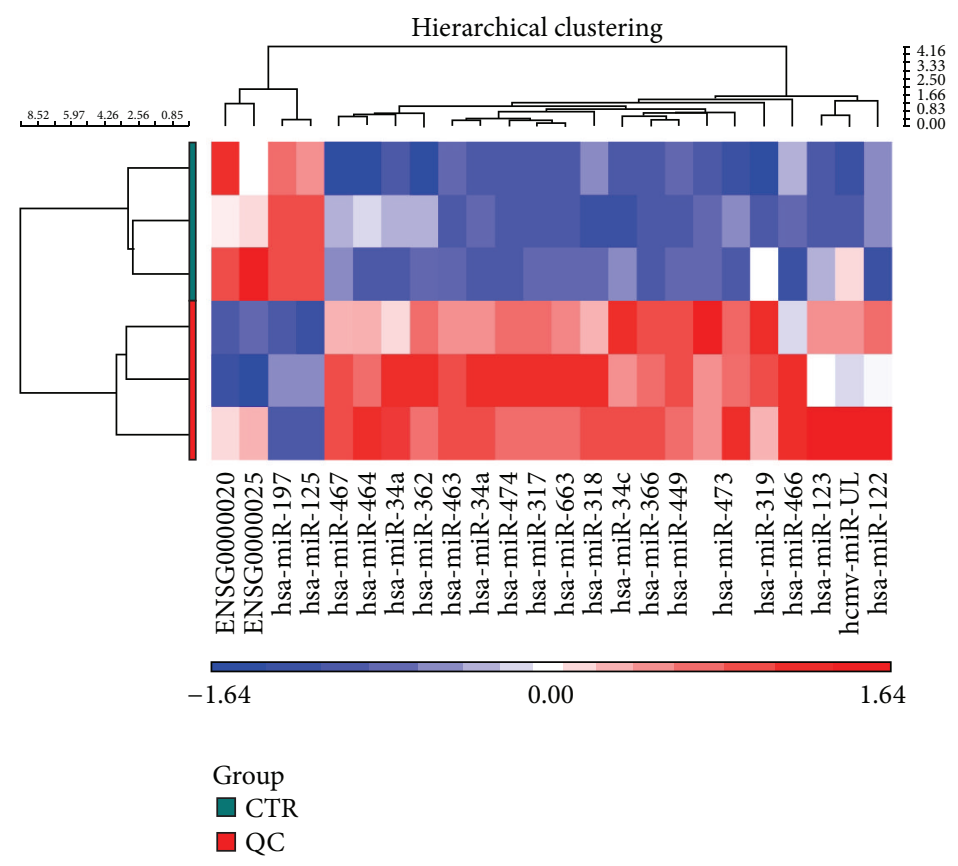

(a)

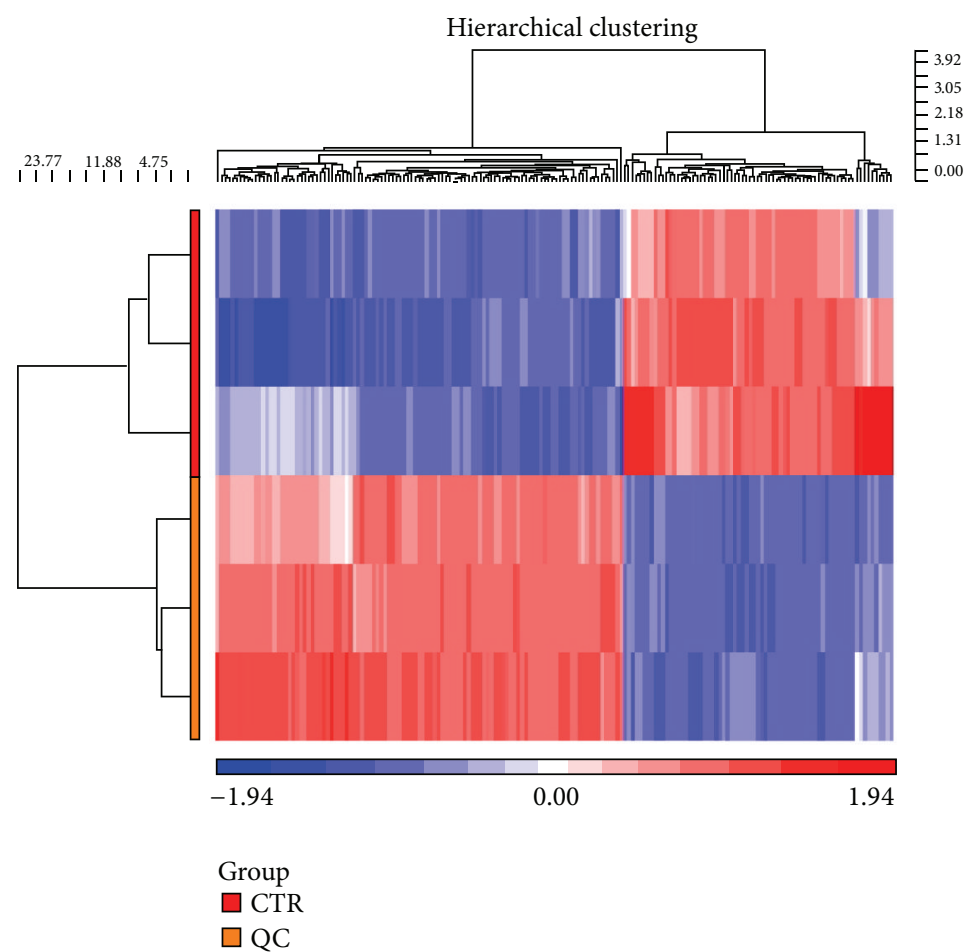

(b)

FIGURE 3: Cluster analysis of differentially expressed miRNA and mRNA with and without QC treatment. (a) miRNA signature in BPH-1 cells treated with QC and controls. (b) mRNA signature in BPH-1 cells treated with QC and controls. Signal intensity was expressed as a log 2 ratio between QC treatment and controls. Bright blue, underexpression; white, no change; bright red, overexpression. $P<0.05$.

\section{Discussion}

Qianliening capsule (QC) is a traditional Chinese formulation that has long been used to clinically treat benign prostate hyperplasia $(\mathrm{BPH})$. However, $\mathrm{BPH}$ is a complex disease as its pathogenesis and progression are associated with multiple factors, genes, and signal transduction pathways, all of which are further highly regulated by an miRNA regulatory network. As such, much effort has been placed on understanding the mechanisms underlying the disease process and 


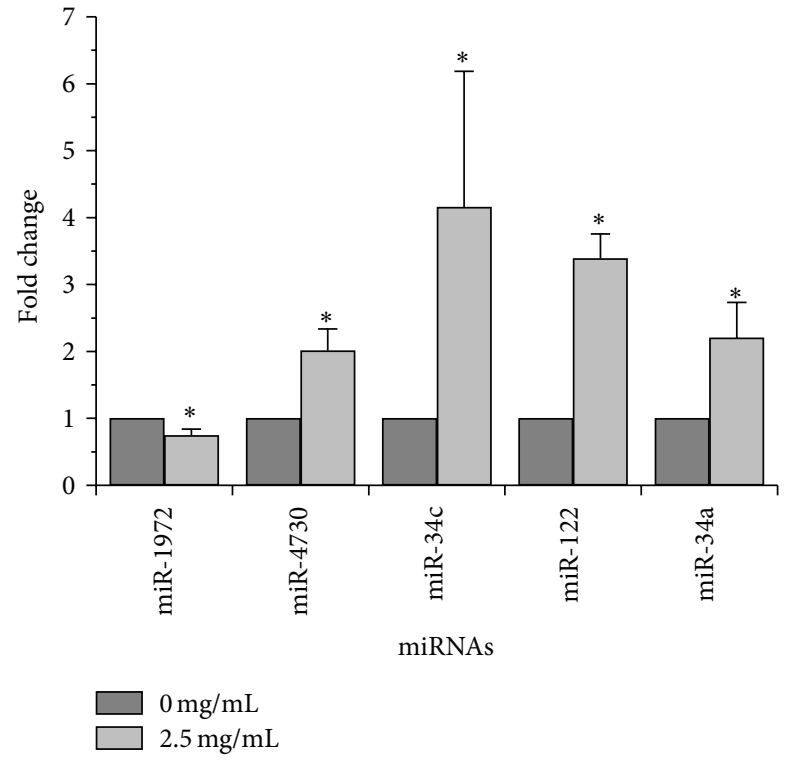

(a)

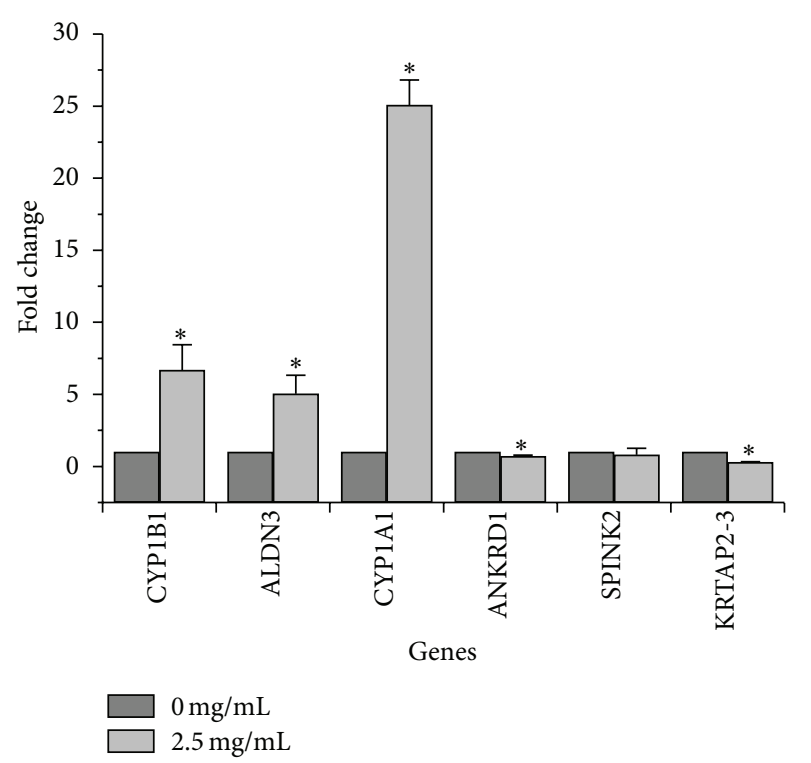

(b)

FIGURE 4: Confirmation of miRNA and mRNA expression by real-time PCR. (a) The miRNA levels of different miRNA in QC-treated and untreated cells were determined by real-time PCR. (b) The mRNA levels of different mRNA in QC-treated and untreated cells were determined by real-time PCR. U6 was used as the internal controls for the real-time PCR, respectively. Images are representative from 3 independent cellbased experiments.

TABLE 3: List of enriched KEGG pathways of differentially expressed mRNAs in BPH-1 cells treated with QC $(2.5 \mathrm{mg} / \mathrm{mL})$ and control cells.

\begin{tabular}{lccc}
\hline Pathway ID & Definition & Gene count & $P$ value \\
\hline hsa00980 & Metabolism of xenobiotics by cytochrome P450 & 10 & $1.87 E-09$ \\
hsa00140 & Steroid hormone biosynthesis & 6 & $5.04 E-05$ \\
has00982 & Drug metabolism & 5 & 0.00223 \\
hsa04350 & TGF-beta signaling pathway & 4 & 0.04332 \\
\hline
\end{tabular}

Enriched KEGG pathways were used for analysis of the differentially expressed mRNAs between two groups; $P$ values after multiple testing corrections $<0.05$.

treatment mechanisms of QC in BPH. Research on miRNAs in various diseases is still in its infancy, although exciting findings including the link between miRNAs and prostate cancer have recently been reported [40]. To our knowledge, however, there have been no reports on the association between miRNAs and any spectrum of BPH. In order to identify potentially unique miRNA expression profiles, we jointly employed miRNA and gene microarray analysis to detect aberrantly expressed miRNA and mRNA in BPH-1 cells.

Hundreds of miRNAs have been shown to play important roles in regulating gene expression through degradation of mRNA or repression of translation in a variety of model systems [41, 42]. Microarray gene expression profiling has improved our understanding of BPH biology and allowed the development of multigene "signatures" to predict outcome and response to systemic therapies [32]. Through comprehensive array profiling and analysis of miRNA and gene expression levels, we have identified putative candidate genes and pathways that mediate QCs effects in BPH. Genes differentially expressed in response to QC treatment included those involved in cell growth, proliferation, and apoptosis, common characteristics in hyperplasic diseases.
Our preliminary research showed that QC could inhibit proliferation and promoted apoptosis in vivo and in vitro $[20,21]$. According to our miRNA array results, most of the miRNAs have previously been shown to be associated with apoptosis, proliferation, and metabolism. For example, the miR-34 family, including miR-34a and c, has been known to regulate several cellular events, including the cell cycle, cell migration, and apoptosis [29,30]. miR-663 is involved in the TGF- $\beta$ signaling pathway $[32,33]$. miR-3185 likely plays an important role in regulating MAPK signaling [31]. miR-122 is suggested to regulate many target genes in lipid and cholesterol metabolism [35, 36]. Interestingly, among 242 altered genes identified by our gene microarray experiment, more than 30 genes were involved in apoptosis and proliferation. Their cellular functions encompass regulation of cell proliferation, cell cycle, and cell death. More than 10 genes were involved in metabolism (metabolism of xenobiotics and drug metabolism). The miRNA-mRNA interaction network analysis further integrated bioinformatic findings and then outlined the primary different miRNAs and their major target gene(s). It is of great interest to determine in future studies whether these same sets of miRNAs and their target genes 
TABLE 4: List of enriched GOs of differentially expressed mRNAs in BPH-1 cells treated with QC (2.5 mg/mL) and control cells.

\begin{tabular}{|c|c|c|c|}
\hline ID & Definition & Gene count & $P$ value \\
\hline GO:0055114 & Oxidation reduction & 18 & $1.20 E-06$ \\
\hline GO:0042127 & Regulation of cell proliferation & 15 & $8.27 E-04$ \\
\hline GO:0006955 & Immune response & 14 & $7.61 E-04$ \\
\hline GO:0042981 & Regulation of apoptosis & 14 & 0.002994 \\
\hline GO:0043067 & Regulation of programmed cell death & 14 & 0.00326 \\
\hline GO:0010941 & Regulation of cell death & 14 & 0.003365 \\
\hline GO:0008219 & Cell death & 12 & 0.009416 \\
\hline GO:0016265 & Death & 12 & 0.009889 \\
\hline GO:0012501 & Programmed cell death & 11 & 0.00846 \\
\hline GO:0010033 & Response to organic substance & 11 & 0.024364 \\
\hline GO:0043065 & Positive regulation of apoptosis & 10 & 0.002571 \\
\hline GO:0043068 & Positive regulation of programmed cell death & 10 & 0.002694 \\
\hline GO:0010942 & Positive regulation of cell death & 10 & 0.002778 \\
\hline GO:0009611 & Response to wounding & 10 & 0.009872 \\
\hline GO:0006915 & Apoptosis & 10 & 0.021064 \\
\hline GO:0030182 & Neuron differentiation & 9 & 0.009842 \\
\hline GO:0042493 & Response to drug & 8 & $6.67 E-04$ \\
\hline GO:0044092 & Negative regulation of molecular function & 8 & 0.007664 \\
\hline GO:0022403 & Cell cycle phase & 8 & 0.022599 \\
\hline GO:0008284 & Positive regulation of cell proliferation & 8 & 0.022599 \\
\hline
\end{tabular}

Enriched GOs were used for analysis of the differentially expressed mRNAs between two groups; $P$ values after multiple testing corrections $<0.05$.

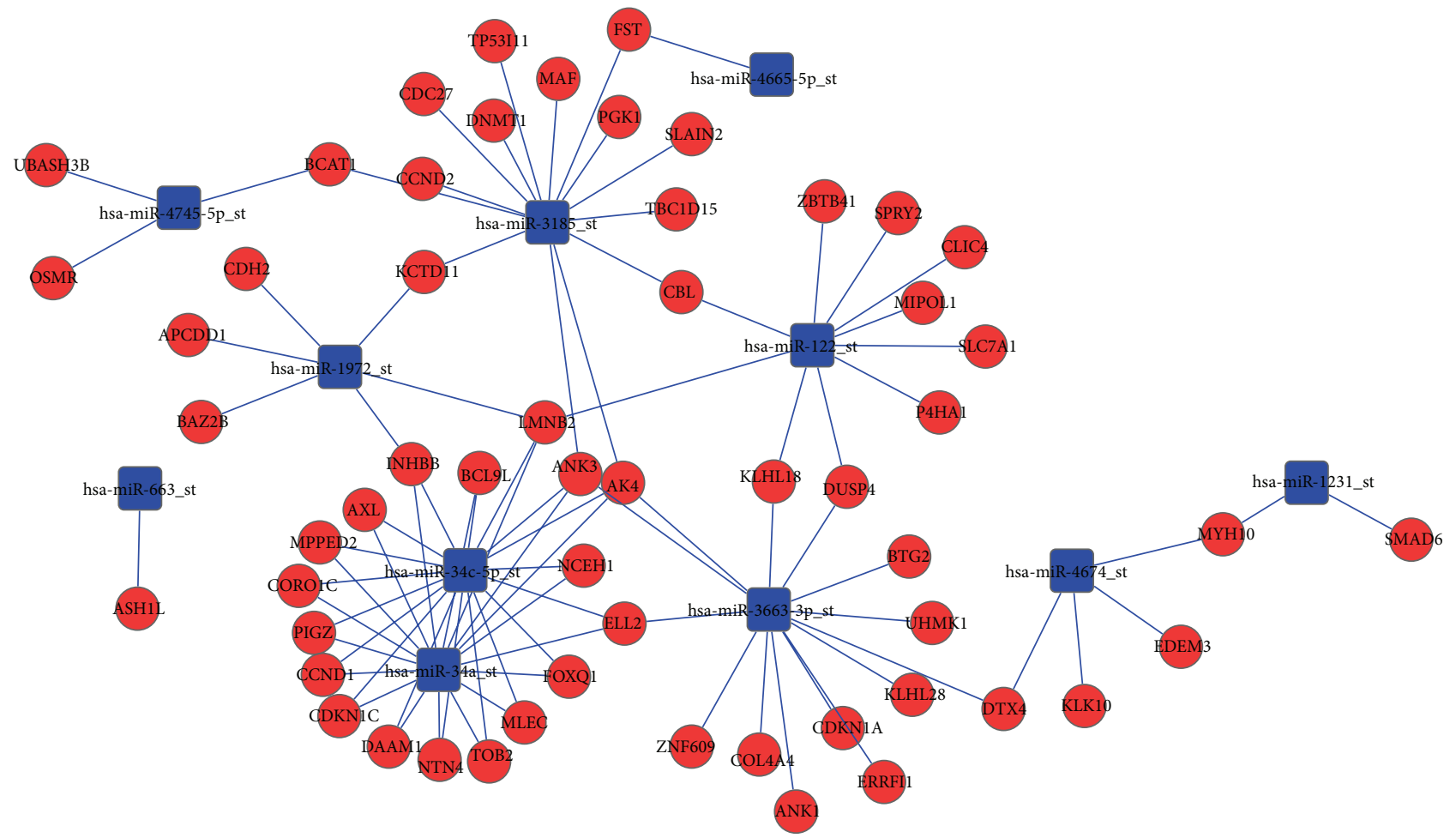

FIGURE 5: Interaction network of upregulated genes and downregulated miRNAs/mRNAs in BPH-1 cells treated with QC (2.5 mg/mL) and control cells. Blue box nodes represent miRNA, and red cycle nodes represent mRNA. Edges show the inhibitory effect of miRNA on mRNA. The miRNA-mRNA network was visualized by Cytoscape tool. 
could be biomarkers that are associated with mechanisms by which QC treats BPH.

\section{Abbreviations \\ BPH: Benign prostatic hyperplasia \\ QC: Qianliening capsule \\ miRNAs: MicroRNAs.}

\section{Conflict of Interests}

The authors declare that there is no conflict of interests regarding the publication of this paper.

\section{Acknowledgments}

This work was sponsored by the National Natural Science Foundation of China (81273928 and 81373817). The authors would like to thank Clarity Manuscript Consultants LLC Indianapolis for technical assistance in editing this paper.

\section{References}

[1] D. R. Paolone, "Benign prostatic hyperplasia," Clinics in Geriatric Medicine, vol. 26, no. 2, pp. 223-239, 2010.

[2] B. Djavan, "Lower urinary tract symptoms/benign prostatic hyperplasia: fast control of the patient's quality of life," Urology, vol. 62, no. 3, pp. 6-14, 2003.

[3] N. C. Lau, L. P. Lim, E. G. Weinstein, and D. P. Bartel, "An abundant class of tiny RNAs with probable regulatory roles in Caenorhabditis elegans," Science, vol. 294, no. 5543, pp. 858-862, 2001.

[4] V. Ambros, "The functions of animal microRNAs," Nature, vol. 431, no. 7006, pp. 350-355, 2004.

[5] J. Brennecke, D. R. Hipfner, A. Stark, R. B. Russell, and S. M. Cohen, "bantam encodes a developmentally regulated microRNA that controls cell proliferation and regulates the proapoptotic gene hid in Drosophila," Cell, vol. 113, no. 1, pp. 2536, 2003.

[6] X. B. Shi, L. Xue, J. Yang et al., "An androgen-regulated miRNA suppresses Bakl expression and induces androgen-independent growth of PCa cells," Proceedings of the National Academy of Sciences of the United States of America, vol. 104, pp. 1998319988, 2007.

[7] A. Musiyenko, V. Bitko, and S. Barik, "Ectopic expression of miR-126*, an intronic product of the vascular endothelial EGFlike 7 gene, regulates prostein translation and invasiveness of prostate cancer LNCaP cells," Journal of Molecular Medicine, vol. 86, no. 3, pp. 313-322, 2008.

[8] Z. Lu, M. Liu, V. Stribinskis et al., "MicroRNA-21 promotes cell transformation by targeting the programmed cell death 4 gene," Oncogene, vol. 27, no. 31, pp. 4373-4379, 2008.

[9] L. Vrba, T. J. Jensen, J. C. Garbe et al., "Role for DNA methylation in the regulation of miR-200c and miR-141 expression in normal and cancer cells," PLoS ONE, vol. 5, no. 1, Article ID e8697, 2010.

[10] E. R. Barrack and S. J. Berry, "DNA synthesis in the canine prostate: effects of androgen and estrogen treatment," Prostate, vol. 10, no. 1, pp. 45-56, 1987.
[11] C. Angelucci, F. Iacopino, G. Lama et al., "Reverse transcriptasePCR analysis of apoptosis-regulating gene expression in human benign prostatic hyperplasia," Anticancer Research, vol. 25, no. 6, pp. 3937-3941, 2005.

[12] M. C. Pollan, H. A. Benghuzzi, and M. Tucci, "Growth factor expression in early stages of benign prostatic hyperplasia upon exposure to sustained delivery of androgens," Biomedical Sciences Instrumentation, vol. 39, pp. 329-334, 2003.

[13] C. G. Roehrborn, "Male lower urinary tract symptoms (LUTS) and benign prostatic hyperplasia (BPH)," Medical Clinics of North America, vol. 95, no. 1, pp. 87-100, 2011.

[14] A. Tiwari, N. S. Krishna, K. Nanda, and A. Chugh, "Benign prostatic hyperplasia: an insight into current investigational medical therapies," Expert Opinion on Investigational Drugs, vol. 14, no. 11, pp. 1359-1372, 2005.

[15] J. Zhou, J. Lin, W. Xu, X. Zhong, J. Xie, and Z. Hong, "Effects of Qianliening capsule on the expression of IL-10 and TNF- $\alpha$ in benign prostatic hyperplasia," Chinese Archives of Traditional Chinese Medicine, vol. 28, pp. 2657-2569, 2010.

[16] J. H. Zhou, Z. F. Hong, J. M. Lin, J. Y. Zhao, and H. T. Zhou, "Effect of Qianliening granule on experimental hyperplasia of prostate," Journal of Fujian University of Traditional Chinese Medicine, vol. 18, pp. 45-47, 2008.

[17] H. T. Zhou, J. M. Lin, J. Y. Zhao, J. H. Zhou, and Z. F. Hong, "Inhibition effects of qianliening granule on IL-1 $\beta$ and its mRNA expression in model rats," Fujian University of Traditional Chinese Medicine, vol. 20, pp. 21-24, 2010.

[18] J. Zhou, J. Lin, X. Wei et al., "Qianliening capsule treats benign prostatic hyperplasia through regulating the expression of sex hormones, estrogen receptor and androgen receptor," African Journal of Pharmacy and Pharmacology, vol. 6, no. 3, pp. 173$180,2012$.

[19] X. Zhong, J. Lin, J. Zhou, W. Xu, Z. Hong, and J. Peng, “Qianliening capsule treats benign prostatic hyperplasia (BPH) by down-regulating the expression of PCNA, CyclinD1 and CDK4," African Journal of Biotechnology, vol. 11, no. 30, pp. 77317737, 2012.

[20] J. Lin, J. Zhou, X. Zhong, Z. Hong, and J. Peng, "Inhibition of the signal transducer and activator of transcription 3 signaling pathway by Qianliening capsules suppresses the growth and induces the apoptosis of human prostate cells," Molecular Medicine Reports, vol. 11, no. 3, pp. 2207-2214, 2015.

[21] J. Zhou, J. Lin, L. Liu, Y. Zheng, and Z. Hong, "Qianliening capsules influence the apoptosis of benign prostatic hyperplasia epithelial-1 cells by regulating the extracellular matrix," Molecular Medicine Reports, vol. 11, no. 5, pp. 3734-3740, 2015.

[22] C. Chen, D. A. Ridzon, A. J. Broomer et al., "Real-time quantification of microRNAs by stem-loop RT-PCR," Nucleic Acids Research, vol. 33, no. 20, article el79, 2005.

[23] F. Tang, P. Hajkova, S. C. Barton, K. Lao, and M. A. Surani, "MicroRNA expression profiling of single whole embryonic stem cells," Nucleic Acids Research, vol. 34, no. 2, article e9, 2006.

[24] K. J. Livak and T. D. Schmittgen, "Analysis of relative gene expression data using real-time quantitative PCR and the $2^{-\Delta \Delta C}$ T method," Methods, vol. 25, no. 4, pp. 402-408, 2001.

[25] M. Kanehisa, S. Goto, M. Furumichi, M. Tanabe, and M. Hirakawa, "KEGG for representation and analysis of molecular networks involving diseases and drugs," Nucleic Acids Research, vol. 38, no. 1, pp. D355-D360, 2009.

[26] T. Zhang, M. Jiang, L. Chen, B. Niu, and Y. Cai, "Prediction of gene phenotypes based on GO and KEGG pathway enrichment 
scores," BioMed Research International, vol. 2013, Article ID 870795, 7 pages, 2013.

[27] Z. Yi, J. Li, K. Gao, and Y. Fu, "Identifcation of differentially expressed long non-coding RNAs in CD4+ T cells response to latent tuberculosis infection," Journal of Infection, vol. 69, no. 6, pp. 558-568, 2014.

[28] P. Shannon, A. Markiel, O. Ozier et al., "Cytoscape: a software Environment for integrated models of biomolecular interaction networks," Genome Research, vol. 13, no. 11, pp. 2498-2504, 2003.

[29] S. Roy, E. Levi, A. P. Majumdar, and F. H. Sarkar, "Expression of miR-34 is lost in colon cancer which can be re-expressed by a novel agent CDF,' Journal of Hematology and Oncology, vol. 5, article 58, 2012.

[30] O. A. Ebner and M. Selbach, "Quantitative proteomic analysis of gene regulation by miR-34a and miR-34c," PLoS ONE, vol. 9, no. 3, Article ID e92166, 2014.

[31] C. Long, L. Jiang, F. Wei et al., "Integrated miRNA-mRNA analysis revealing the potential roles of miRNAs in chordomas," PLoS ONE, vol. 8, no. 6, Article ID e66676, 2013.

[32] Y. Zhang, X. Zhou, X. Xu et al., "Waltonitone induces apoptosis through mir-663-induced Bcl-2 downregulation in non-small cell lung cancer," Tumor Biology, vol. 36, no. 2, pp. 871-876, 2015.

[33] Q. Hong, S. Yu, X. Geng et al., "High concentrations of uric acid inhibit endothelial cell migration via miR-663 which regulates PTEN by targeting TGF- $\beta 1$," Microcirculation, vol. 22, no. 4, pp. 306-314, 2015.

[34] L. Zhang, Y. Ge, and E. Fuchs, " $m i R-125 b$ can enhance skin tumor initiation and promote malignant progression by repressing differentiation and prolonging cell survival," Genes \& Development, vol. 28, no. 22, pp. 2532-2546, 2014.

[35] C. Esau, S. Davis, S. F. Murray et al., "miR-122 regulation of lipid metabolism revealed by in vivo antisense targeting," Cell Metabolism, vol. 3, no. 2, pp. 87-98, 2006.

[36] A. P. Lewis and C. L. Jopling, "Regulation and biological function of the liver-specific miR-122," Biochemical Society Transactions, vol. 38, no. 6, pp. 1553-1557, 2010.

[37] C. Zhou, Q. Yu, L. Chen, J. Wang, S. Zheng, and J. Zhang, "A miR-1231 binding site polymorphism in the 3'UTR of IFNAR1 is associated with hepatocellular carcinoma susceptibility," Gene, vol. 507, no. 1, pp. 95-98, 2012.

[38] M. Sand, M. Skrygan, D. Sand et al., "Comparative microarray analysis of microRNA expression profiles in primary cutaneous malignant melanoma, cutaneous malignant melanoma metastases, and benign melanocytic nevi," Cell and Tissue Research, vol. 351, no. 1, pp. 85-98, 2013.

[39] M. M. Perry, E. Tsitsiou, P. J. Austin et al., "Role of non-coding RNAs in maintaining primary airway smooth muscle cells," Respiratory Research, vol. 15, no. 1, article 58, 2014.

[40] H.-L. Zhang, X.-J. Qin, D.-L. Cao et al., "An elevated serum miR-141 level in patients with bone-metastatic prostate cancer is correlated with more bone lesions," Asian Journal of Andrology, vol. 15, no. 2, pp. 231-235, 2013.

[41] D. P. Bartel, "MicroRNAs: genomics, biogenesis, mechanism, and function," Cell, vol. 116, no. 2, pp. 281-297, 2004.

[42] M. Lagos-Quintana, R. Rauhut, W. Lendeckel, and T. Tuschl, "Identification of novel genes coding for small expressed RNAs," Science, vol. 294, no. 5543, pp. 853-858, 2001. 


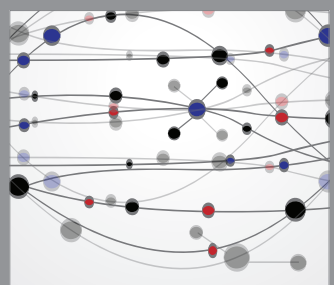

The Scientific World Journal
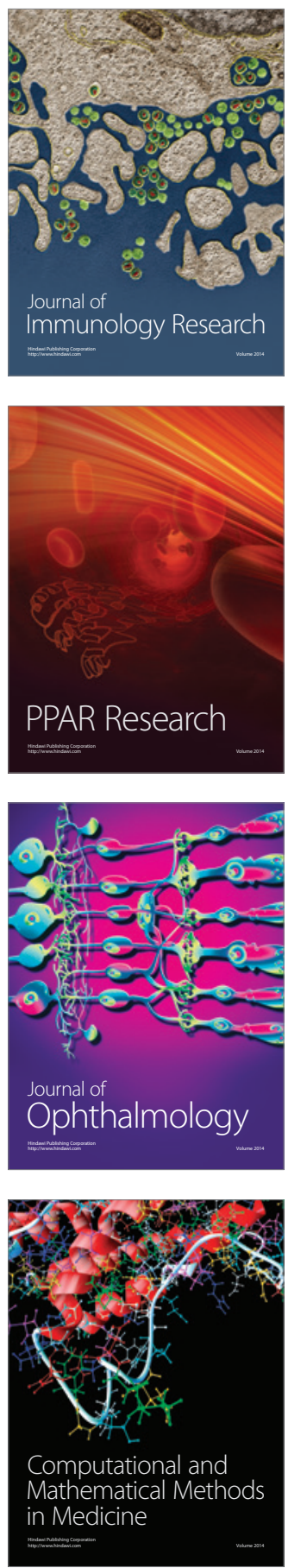

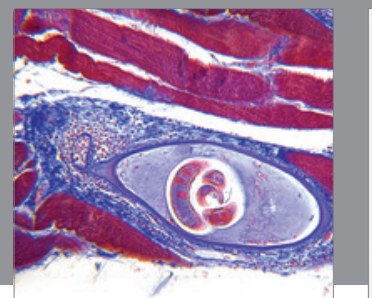

Gastroenterology

Research and Practice
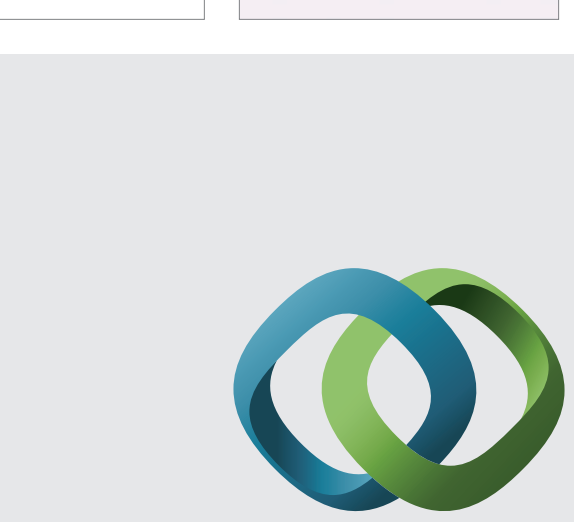

\section{Hindawi}

Submit your manuscripts at

http://www.hindawi.com
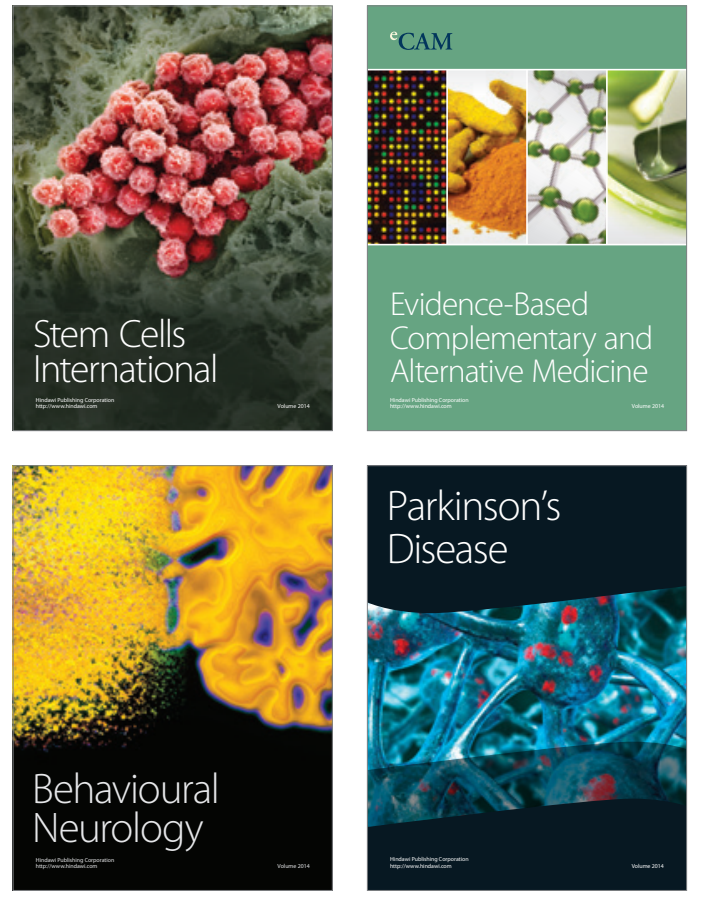
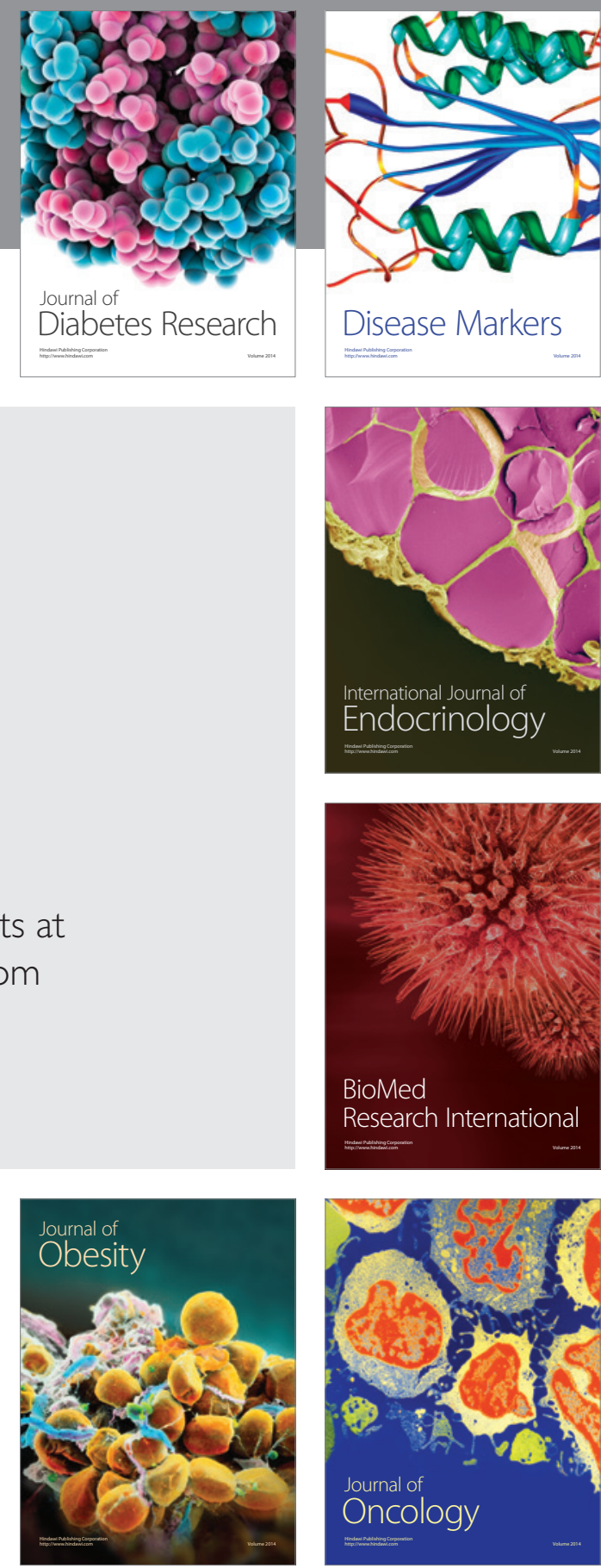

Disease Markers
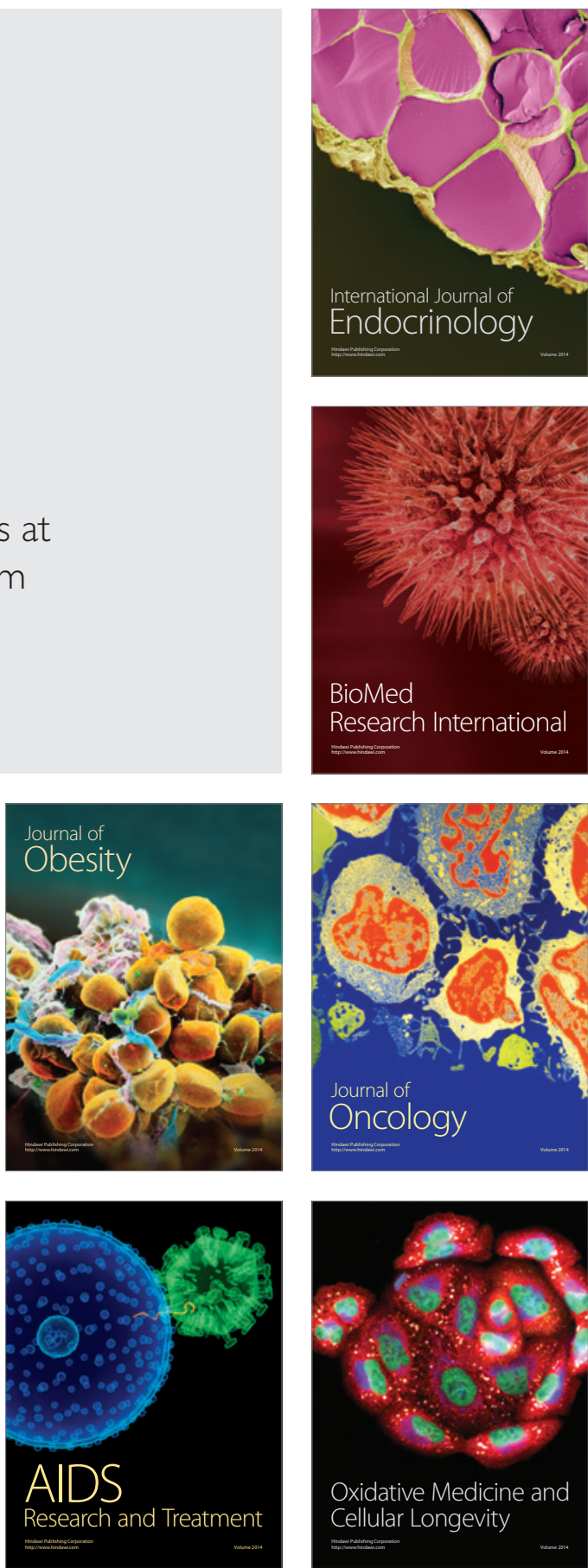